\title{
Concise review of the coronavirus disease 2019 outbreak
}

\author{
Gloria M. Aguirre-García, Jaime E. Espinosa-Mora, Javier Ramos-Jiménez, and Adrián Camacho-Ortiz* \\ Infectious Diseases Service, "Dr. José Eleuterio González" University Hospital and School of Medicine, Universidad Autónoma de Nuevo León, \\ Monterrey, Nuevo Leon, Mexico
}

\begin{abstract}
Coronaviruses have been well known for causing common cold syndrome in healthy people. Two beta-coronaviruses that emerged from animal reservoirs, severe acute respiratory syndrome coronavirus (SARS-CoV), and Middle East respiratory syndrome coronavirus caused global epidemics in 2002 and 2012. In December 2019, a cluster of pneumonia cases in the city of Wuhan in the province of Hubei, China, with an unknown etiology was reported by Chinese authorities. The full genomic sequence of the novel coronavirus was released by January 2020 and it was designated the 2019 novel coronavirus by the World Health Organization (WHO). Later, the International Committee on the Taxonomy of Viruses named it SARS-CoV-2 and the WHO designated this new disease as COVID-19. By June 22, 2020, there were 8,860,331 confirmed cases globally, and a total of 465,740 deaths. At present, there is no approved vaccine against SARS-CoV-2 and no specific medication for treatment, although some drugs have shown some in vitro activity and promising outcomes regarding mortality in recent clinical trials. As of today, the most effective mechanism in prevention is avoidance of exposure and basic respiratory hygiene. Caution is warranted due to the great amount of available information rapidly obtained, and the fact that there is scientific uncertainty. Answers will be found with well-designed studies that require time and patience.
\end{abstract}

Key words: Severe acute respiratory syndrome coronavirus-2. Outbreak. Coronavirus disease-19.

\section{Background}

Coronaviruses have been well known for causing common cold syndrome in healthy people. They are large and enveloped RNA positive viruses divided into four genera: alpha, beta, delta, and gamma. Human coronavirus (HCoV) 229E, NL63, OC43, and HKU1 are endemic $\mathrm{HCoV}$ that produce upper respiratory tract infections, which are often mild ${ }^{1}$. Nevertheless, two beta-coronaviruses that emerged from animal reservoirs, severe acute respiratory syndrome (SARS-CoV), and Middle East respiratory syndrome coronavirus (MERS-CoV) caused global epidemics in 2002 and 2012, respectively, with mortality rates of $10 \%$ for SARS-CoV and $37 \%$ for MERS-CoV².

\section{COVID-19 outbreak}

In December 2019, a cluster of pneumonia cases in the city of Wuhan in the province of Hubei, China, with an unknown etiology was reported by Chinese authorities, many of them with exposure to the Huanan seafood wholesale market, where a large variety of animal species were traded. By January 10, 2020, the Shanghai Public Health Clinical Center \& School of Public Health released the full genomic sequence of the novel coronavirus, and it was designated as the 2019 novel coronavirus (2019-nCoV) by the World Health Organization (WHO). A real-time polymerase chain reaction diagnostic test was rapidly developed and enabled confirmation of suspected cases $^{3}$. On January 30, 2020, the WHO's

\footnotetext{
Correspondence:

*Adrián Camacho-Ortiz

E-mail: acamacho_md@yahoo.com

Available online: $30-10-2020$

Date of acceptance: 11-03-2020

Date of reception: 02-06-2020 DOI: 10.24875/RMU.20000038

Medicina Universitaria. 2020;22(3):107-112 www.medicinauniversitaria.org

1665-5796/C 2020 Universidad Autónoma de Nuevo León. Published by Permanyer. This is an open access article under the CC BY-NC-ND license (http://creativecommons.org/licenses/by-nc-nd/4.0/).
} 
emergency committee declared it a Public Health Emergency of International Concern ${ }^{4}$. The Coronavirus Study Group of the International Committee on the Taxonomy of Viruses, responsible of the classification of viruses and the taxonomy of the Coronaviridae family, designated the named 2019-nCoV as Severe Acute Respiratory Syndrome Coronavirus 2 (SARS-CoV-2) on February $11,2020^{5}$ and the WHO announced "COVID-19" as the name of this new disease. On March 11, the WHO declared the COVID-19 outbreak a global pandemic. By June 22, 2020, there were 8,860,331 confirmed cases globally, and a total of 465,740 deaths ${ }^{6}$. Some unprecedented containment strategies have emerged in the past months, such as a regional quarantine affecting around 50 million people in China, travel restrictions and closure of schools and public places in Lombardy, and other provinces in Italy ${ }^{7}$, whole cruise ships held in quarantine in ports and regulation policies for travelers from all around the world.

\section{Transmission}

The majority of the earliest cases of the SARS-CoV-2 were associated with exposure to the Huanan seafood wholesale market. However, human-to-human transmission was documented, as reported by Chan et al. ${ }^{8}$, as well as the infection cases of 15 health-care practitioners in a Wuhan hospital ${ }^{3}$. Human-to-human transmission occurs mainly through respiratory droplets, similar to how influenza and other respiratory viruses spread. Reports of viral transmission by asymptomatic carriers surfaced in Germany ${ }^{9}$ and in a familial cluster in Anyang, China ${ }^{10}$. To estimate the transmission potential of this disease, the reproduction number $\left(R_{0}\right)$ has been widely used as a measure of viral spread. It represents the expected number of secondary infectious cases resulting from a primary infectious case in a susceptible population. A value greater than 1 predicts that the number of cases is likely to increase. SARS and MERS had $R_{0}$ of 0.67-1.23 and 0.29-0.80, respectively ${ }^{11}$. In a study by Li et al., the $R_{0}$ has been estimated to be 2.2, which means that each patient can spread the infection to 2.2 other people ${ }^{12}$. Nevertheless, this number can be as high as $3.58^{13}$ and can vary considerably by location, age group, and over time.

\section{Clinical manifestations}

There is growing data regarding the clinical presentation of patients with COVID-19. The incubation period has been estimated to be as short as 5.1 days (95\% confidence interval $[\mathrm{Cl}], 4.5-5.8)^{14}$. As of January 24, 2020, The Lancet published the clinical features of the first 41 confirmed cases. Underlying diseases were present in less than half of cases, including diabetes in $20 \%$, hypertension in $15 \%$, and cardiovascular disease in $15 \%$ of cases. The most common symptoms at onset were fever in $98 \%$ of patients, cough in $76 \%$, dyspnea in $55 \%$, and myalgias or fatigue in $44 \%$. All cases had abnormal findings on chest computed tomography, and complications as acute respiratory syndrome occurred in $29 \%$ of cases. The mortality rate was $15 \% 2$.

In a most recent descriptive analysis by The Novel Coronavirus Pneumonia Emergency Response Epidemiology Team that included 72,314 patient records and 16,186 confirmed cases, $25.2 \%$ of patients had a comorbid condition and $80.9 \%$ were classified as mild cases. The overall case-fatality rate was $2.3 \%$ of confirmed cases. The $>80$ age group had the highest case fatality rate at $14.8 \%{ }^{15}$.

Emerging information has reported not only respiratory symptoms but also gastrointestinal (Gl), neurological, and cutaneous manifestations. A meta-analysis and systematic review of 60 studies with data on GI symptoms found that pooled prevalence was $18 \%$. The most common symptoms were anorexia, diarrhea, and abdominal pain. Prevalence was higher in patients with severe cases of the disease compared to non-severe disease $(17 \% \text { vs. } 12 \%)^{16}$.

In addition, there is evidence that coronaviruses have a certain tropism for the central nervous system. Neurological manifestations such as acute cerebrovascular disease, impaired consciousness, and skeletal muscle injury have been described in patients with severe COVID-1917.

Anosmia and dysgeusia are symptoms often dismissed but that appear early in the disease course. In a cross-sectional study made in Italy, $33.9 \%$ of hospitalized patients with confirmed SARS-CoV-2 infection self-reported at least one taste or olfactory disorder and $18.6 \%$ reported both ${ }^{18}$.

Therefore, clinical presentation varies widely among individuals and might be more severe in specific populations such as elderly patients and those with preexisting comorbidities.

\section{Diagnosis}

On May 6, 2020, the Infectious Diseases Society of America published guidelines on the diagnosis of COVID-19. It recommends a SARS-CoV-2 nucleic acid amplification test in symptomatic individuals with 
clinical suspicion of COVID-19, even if the latter is low. Symptomatic patients should be considered if having respiratory symptoms alone, such as cough or shortness of breath, or at least two of the following symptoms: fever, chills, muscle pain, headache, sore throat, or new loss of taste or smell19. Samples to be collected for the diagnostic purpose include nasopharyngeal and oropharyngeal swab, sputum, if produced, and in patients with more severe respiratory disease, endotracheal aspirate, or bronchoalveolar lavage ${ }^{20}$. Guidelines suggest the collection of nasopharyngeal, mid-turbinate, or nasal swabs rather than oropharyngeal swabs or saliva because sensitivity from the former is higher, ranging from $76 \%$ to $100 \%$, and with $100 \%$ specificity ${ }^{19}$. Nevertheless, some studies have demonstrated the sensitivity and feasibility of collecting saliva as an alternative. Williams et al. reported that 33 of $35(85 \%)$ patients had SARS-CoV-2 detected in saliva, though the $\mathrm{Ct}$ value was significantly lower in nasopharyngeal swabs compared to saliva, showing higher viral loads in nasopharyngeal specimens ${ }^{21}$.

Co-infections have been reported, so all patients should be considered for testing for other respiratory viruses. The most common co-infections in a study of 1217 specimens, with $7.5 \%$ of them positive for SARS-CoV-2 and additional pathogens, were rhinovirus/enterovirus $(6.9 \%)$, respiratory syncytial virus $(5.2 \%)$, and non-SARS-CoV-2 Coronaviridae $(4.3 \%)^{22}$.

Serology tests are additional tools that have been used for the detection of seroconversion in patients infected with SARS-CoV-2. Rates of seroconversion reported by Zhao et al. were $93.1 \%$ for total antibodies, which were $8.27 \%$ for immunoglobulin $M$ and $64.7 \%$ for immunoglobulin $\mathrm{G}$ with a median time of 11,12 , and 14 days, respectively ${ }^{23}$. In Santa Clara County, California, Bendavid et al. found that the COVID-19 seroprevalence was $1.5 \%$ and adjusted prevalence was $2.81 \%^{24}$. Many commercial serological rapid tests are circulating but not all of them have been validated by the Food and Drug Administration (FDA). Although their diagnostic performance is limited, they might be helpful in determining the immune response in certain areas and populations.

\section{Prevention measures}

At present, there are more than 10 vaccine candidates in clinical evaluation, and one of them is being assessed in a phase 3 trial. Despite this, no approved vaccine is available yet. Effective preventive measures should still be implemented to reduce the risk of transmission of SARS-CoV-2.

The most effective mechanism in prevention is avoidance of exposure. Standard precautions such as ${ }^{20}$ :

- Frequent hand washing with soap and water or alcohol-based solutions containing at least $60 \%$ alcohol.

- Avoid facial contact with unwashed hands and close contact with people who are sick. It is known that the virus has the ability to infect from a distance of $2 \mathrm{~m}$. In case of sneezing or coughing, cover with a tissue and immediately deposit it in the trash can. In the case of no tissue, the elbow should be used

- Wash surfaces and frequently contacted objects.

- Avoid close contact with people who are sick, even inside your home

All the measures mentioned above apply to any type of population and have helped to reduce the risk of infection with respiratory viruses, so these are actions that must be implemented daily and become habits. However, health workers are a population at high risk of infection and other measures must be taken within hospitals to prevent the spread of infection ${ }^{20}$ (Table 1).

\section{Treatment}

There is no specific medication for SARS-CoV-2. Management includes isolation measures to prevent the spread of infection and supportive therapy for those who might have the disease ${ }^{20}$. Early identification as well as the implementation of preventive measures is the key to stop the spread of germs ${ }^{25}$.

Protease inhibitors such as lopinavir and ritonavir, used for HIV treatment, were used in combination among patients with SARS-CoV, showing clinical benefits and fewer adverse clinical outcomes. In 2004, in vitro activity against SARS-CoV was demonstrated with lopinavir/ritonavir and ribavirin. These patients showed a reduction in viral load and appeared to have a milder disease course $^{26}$. In Saudi Arabia, the combination of lopinavir/ ritonavir with recombinant interferon-B1b has been used for the treatment of MERS-CoV to evaluate mortality compared to placebo. A randomized clinical trial with 13 patients already enrolled has begun ${ }^{27}$. Other antivirals and drugs such as remdesivir and chloroquine have shown in vitro activity, and human trials are running ${ }^{28}$.

Antimalarial drugs, chloroquine, and hydroxychloroquine are used in the treatment of patients with COVID-19 because it seems to block the virus entry into the host cell, decrease viral replication, and shedding and have anti-inflammatory actions. However, the exact mechanism by which these drugs work against 
Table 1. Preventive measures to limit the spread of COVID-19 in hospital settings

Preventive measures to limit the spread of COVID-19 in hospital settings

a) Any patient with suspected COVID-19 must be put into respiratory, contact, and airborne isolation. In addition, patients must be in an area away from other patients.

b) Limit the exposure time of a suspicious patient with others. If this cannot be done, an N95 facemask must be immediately placed.

c) Any person in contact with the suspected patient must wear an N95 facemask, eye protection, and a protective suit provided by the institution.

d) Apply the general measures, mentioned above, for all patients.

e) In case of no isolated rooms, all patients suspected of COVID-19 must be together at a minimum distance of $1 \mathrm{~m}$.

f) Handwashing with water and soap after removal of protective suit and facemask, once contact with the patient is finished, is required.

g) Avoid unnecessary transfer of patients out of the room. If required, determine a route with the preventive measures previously mentioned.

COVID-19 is speculative. In vitro studies showed that hydroxychloroquine's inhibitory effects were more potent than chloroquine and suggested to treat SARS-CoV-2 infection with a loading dose of $400 \mathrm{mg}$ of hydroxychloroquine sulfate twice daily of to be given orally on day 1 , followed by $200 \mathrm{mg}$ twice daily for 4 more days ${ }^{29}$. Although in vitro studies provide a rationale for its use in COVID-19, there is limited evidence of its clinical efficacy in vivo, and adverse effects such as cardiac complications, QTc interval prolongation, skin reactions, and psychiatric effects should be considered ${ }^{30}$.

Azithromycin was also tested because of its anti-inflammatory effects against SARS-CoV-2 in conjunction with hydroxychloroquine, but current studies found no evidence of strong antiviral activity or clinical benefit in patients with COVID19, and caution must be taken because of their cardiac toxicity. Despite the reported antiviral activity of these two drugs, there is a lack of evidence to treat SARS-CoV-2 infection with these drugs ${ }^{31}$.

Tocilizumab, a humanized immunoglobulin that blocks the interleukin (IL)-6 receptor, is being used due to its antigenic actions against cytokine release syndrome. Previous studies in France have shown that tocilizumab reduced the requirement for mechanical ventilation and reduced the risk of intensive care unit admission. However, there was no change in mortality ${ }^{28}$. It is only recommended in severe cases of COVID-19 with extensive lung disease and elevated IL-6, but it carries an FDA warning of serious infections, including tuberculosis, bacterial, and invasive fungal infections ${ }^{32}$.

Remdesivir, originally developed for use against the Ebola virus, is a novel nucleotide analog prodrug, which is incorporated into nascent viral RNA chains, causing premature termination of RNA transcription.
In vitro studies demonstrated inhibition of SARS-CoV-2 replication and clinical trials are ongoing ${ }^{30}$. Case reports showed promising results, but adverse reactions such as hepatotoxicity, diarrhea, rash, and renal impairment may limit its use. However, Lescure et al. ${ }^{32}$ reported their case series, where three patients received remdesivir, which was well-tolerated, and two patients were discharged after treatment. Recently, the FDA approved the use of remdesivir in emergency situations for SARS-CoV-2 infection, showing a reduction in clinical improvement time and without clinical benefits ${ }^{33}$.

The WHO states that there is currently no evidence for any specific treatment for COVID-19 and investigational therapies should only be used in approved, randomized, and controlled trials.

One therapy that is currently ongoing in clinical trials is convalescent plasma (blood plasma from a person who has recovered from infection and contains neutralizing antibodies against the offending agent). It has been explored as a treatment in SARS and severe influenza, and meta-analysis has noted it may reduce mortality ${ }^{28}$.

Duan et al. ${ }^{30}$ reported ten patients with severe COVID-19 in China who received convalescent plasma in addition to various other treatments. Clinical symptoms improved within 3 days and viral load became undetectable within 6 days of transfusion. Meanwhile, more studies are needed, but preliminary reports in ongoing clinical trials have shown clinical and radiological improvement in patients who have received convalescent plasma ${ }^{30}$.

As this therapy emerges, potential risks include pathogen transmission, allergic transfusion reactions, transfusion-associated circulatory overload, and transfusion-related acute lung injury. In the meantime, the US FDA has listed convalescent plasma as an 
emergency investigational new treatment for patients with serious or immediately life-threatening COVID-1934.

Other antivirals and anti-inflammatory drugs are being considered for COVID-19 treatment in clinical trials such as baricitinib, ruxolitinib, and fedratinib ${ }^{35}$. More recently, a preprint article of the RECOVERY trial was published regarding the effect of dexamethasone in hospitalized patients with COVID-19 and showed reduced 28-day mortality in patients with respiratory support compared to usual care (relative risk [RR] 0.65 $[95 \% \mathrm{Cl} 0.51-0.82, \mathrm{p}<0.001]$ in patients with invasive mechanical ventilation and $\mathrm{RR} 0.80[95 \% \mathrm{Cl} 0.70-0.92$; $p=0.002]$ in patients receiving oxygen without invasive mechanical ventilation). Nonetheless, it must be used cautiously and every case should be individualized ${ }^{36}$.

\section{Clinical prognosis}

The vast majority of patients has mild to moderate symptoms and recovers spontaneously. Mortality has varied from initial reports of $11 \%{ }^{3}$ to subsequent studies of $2.8 \%{ }^{37}$ and the latest report from the $\mathrm{WHO}^{38}$ showing a $5.2 \%$ mortality rate. This will likely continue decreasing as the outbreak is contained. With the available data, COVID-19's fatality rate seems to be significantly lower than other coronavirus outbreaks, such as SARS-CoV with $9.5 \%$ and MERS-CoV with $34.4 \%$, and other viral outbreaks such as influenza A H7N9 with $39 \%$ and the Ebola virus with $63 \% 39$.

\section{Global perspective}

Experience from other outbreaks and pandemics leads to three possible scenarios: (1) that containment and preventive measures limit spread and the number of cases progressively diminishes and gradually disappears as what happened with SARS-CoV-2, (2) after containment, regional transmission occurs, and spikes of activity continue on a local or regional level for months or years, and (3) widespread dissemination continues and the virus becomes endemic in humans, limited by immune response and vaccination.

\section{Areas of uncertainty}

We have to be careful with the great amount of rapidly obtained information on this virus, and the fact that there are many unanswered scientific questions. For example: the origin, the predictions of spread, the proportion of asymptomatic cases, and whether it can recur or infected individuals can be re-infected, as well as the economic impact currently affecting many countries and regions worldwide, and the global burden in the following months and years.

We must consider these and other factors since it is still early in the epidemic, and the answers will only be obtained with scientific well-designed studies that require time and patience.

\section{Conflicts of interest}

The authors have no conflicts of interest to declare.

\section{Funding}

The authors have not declared a specific grant for this research from any funding.

\section{Ethical disclosures}

Protection of human and animal subjects. The authors declare that no experiments were performed on humans or animals for this study.

Confidentiality of data. The authors declare that no patient data appear in this article.

Right to privacy and informed consent. The authors declare that no patient data appear in this article.

\section{References}

1. Paules $\mathrm{Cl}$, Marston HD, Fauci AS. Coronavirus infections-more than just the common cold. JAMA. 2020;323:707-8.

2. Huang C, Wang Y, Li X, Ren L, Zhao J, Hu Y, et al. Clinical features of patients infected with 2019 novel coronavirus in Wuhan, China. Lancet. 2020;395:497-506

3. Wang C, Horby PW, Hayden FG, Gao GF. A novel coronavirus outbreak of global health concern. Lancet. 2020;395:470-3.

4. Statement on the Second Meeting of the International Health Regulations. Emergency Committee Regarding the Outbreak of Novel Coronavirus (2019-nCoV); 2005. Available from: https://www.who.int/news-room/detail/30-01-2020-statement-on-the-second-meeting-of-the-international-health-regulations-(2005)-emergency-committee-regardingthe-outbreak-of-novel-coronavirus-(2019-ncov). [Last accessed on 2020 Feb 05].

5. Gorbalenya AE, Baker SC, Baric RS, De Groot J, Drosten C, Gulyaeva AA, et al. Severe Acute Respiratory Syndrome-Related Coronavirus: The Species and its Viruses-a Statement of the Coronavirus Study Group. New York: bioRxiv; 2020. p. 1-15. [cited 05 Feb 2020]. Available from: https://www.biorxiv.org/content/10.1101/2020.02.07.937862v1.full.

6. World Health Organization. Novel Coronavirus (2019-nCoV). Geneva: World Health Organization; 2020. p. 1-7.

7. Lowen M. Coronavirus: Northern Italy Quarantines 16 Million People. BBC News; 2020. [cited 2 June 2020]. Available from: https://www.bbc. com/news/world-middle-east-51787238.

8. Chan JF, Yuan S, Kok KH, To KK, Chu H, Yang J, et al. A familial cluster of pneumonia associated with the 2019 novel coronavirus indicating person-to-person transmission: a study of a family cluster. Lancet. 2020; 395:514-23.

9. Rothe C, Schunk M, Sothmann P, Bretzel G, Froeschl G, Wallrauch C et al. Transmission of 2019-nCoV infection from an asymptomatic contact in Germany. N Engl J Med. 2020;382:970-1.

10. Bai Y, Yao L, Wei T, Tian F, Jin DY, Chen L, et al. Presumed asymptomatic carrier transmission of COVID-19. JAMA. 2020;323:1406-7.

11. Liu Y, Gayle AA, Wilder-Smith A, Rocklöv J. The reproductive number of COVID-19 is higher compared to SARS coronavirus. J Travel Med. 2020;27:taaa021. 
12. Li Q, Guan X, Wu P, Wang X, Zhou L, Tong $Y$, et al. Early transmission dynamics in Wuhan, China, of novel coronavirus-infected pneumonia. N Engl J Med. 2020;382:1199-207.

13. Zhao S, Lin Q, Ran J, Musa SS, Yang G, Wang W, et al. Preliminary estimation of the basic reproduction number of novel coronavirus (2019 nCoV) in China, from 2019 to 2020: a data-driven analysis in the early phase of the outbreak. Int J Infect Dis. 2020;92:214-7.

14. Lauer SA, Grantz KH, Bi Q, Jones FK, Zheng Q, Meredith HR, et al. The incubation period of coronavirus disease 2019 (COVID-19) from publicly reported confirmed cases: estimation and application. Ann Intern Med. 2020;172:577-82.

15. The Novel Coronavirus Pneumonia Emergency Response Epidemiology Team. The epidemiological characteristics of an outbreak of 2019 novel coronavirus diseases (COVID-19)-China, 2020. Chin Cent Dis Control Prev. 2020;2:113-22.

16. Cheung KS, Hung IF, Chan PP, Lung KC, Tso E, Liu R, et al. Gastroin testinal manifestations of SARS-CoV-2 infection and virus load in fecal samples from a Hong Kong cohort: systematic review and meta-analysis. Gastroenterology. 2020;159:81-95

17. Mao L, Jin $H$, Wang $M, H u Y$, Chen $S, H e ~ Q$, et al. Neurologic manifestations of hospitalized patients with coronavirus disease 2019 in Wuhan China. JAMA Neurol. 2020;77:1-9.

18. Giacomelli A, Pezzati L, Conti F, Bernacchia D, Siano M, Oreni L, et al. Self-reported olfactory and taste disorders in patients with severe acute respiratory coronavirus 2 infection: a cross-sectional study. Clin Infect Dis. 2020;71:889-90.

19. Hanson KE, Caliendo AM, Arias CA, Englund JA, Lee MJ, Patel R, et al. Infectious diseases society of America guidelines on the diagnosis of COVID-19. Clin Infect Dis. 2020; ciaa760.

20. Prevention CDC 2019 Novel Coronavirus; 2020. [cited: 2 June 2020] Available from: https://www.cdc.gov/coronavirus/2019-ncov/about/index.html.

21. Williams E, Bond K, Zhang B, Putland M, Williamson DA. Saliva as a noninvasive specimen for detection of SARS-CoV-2. J Clin Microbiol. 2020;58:e00776-20

22. Kim D, Quinn J, Pinsky B, Shah NH, Brown I. Rates of co-infection between SARS-cov-2 and other respiratory pathogens. J Am Med Assoc. 2020;323:2085-6.

23. Zhao J, Yuan Q, Wang H, Liu W, Liao X, Su Y, et al. Antibody responses to SARS-CoV-2 in patients of novel coronavirus disease 2019. Clin Infect Dis. 2020;???:ciaa344.

24. Bendavid E, Mulaney B, Sood N, Shah S, Ling E, Bromley-Dulfano R, et al COVID-19 Antibody Seroprevalence in Santa Clara County, California New York: medRxiv; 2020. [cited 2 June 2020] Available from: https:// www.medrxiv.org/content/10.1101/2020.04.14.20062463v1?fbclid=IwAR3NrK8oRFFOVulmb1 4hMJpOUvKUgC6MuS7vi7jPvNyy2xeTPIZoaYmlxA

25. Control EC for DP and Novel Coronavirus; 2020. [cited 2 June 2020] Available from: https://www.ecdc.europa.eu/en/novel-coronavirus-china.
26. Chu CM, Cheng VC, Hung IF, Wong MM, Chan KH, Chan KS, et al. Role of lopinavir/ritonavir in the treatment of SARS: initial virological and clinical findings. Thorax. 2004;59:252-6.

27. Arabi YM, Alothman A, Balkhy HH, Al-Dawood A, AlJohani S, Al Harbi S, et al. Treatment of Middle East respiratory syndrome with a combination of lopinavir-ritonavir and interferon- $\beta 1 \mathrm{~b}$ (MIRACLE trial): study protocol for a randomized controlled trial. Trials. 2018;19:81.

28. Roumier M, Paule R, Groh M, Vallee A, Ackermann F. Interleukin-6 Blockade for Severe COVID-19. New York: medrxiv; 2020.

29. Mair-Jenkins J, Saavedra-Campos M, Baillie JK, Cleary P, Khaw FM, Lim WS, et al. The effectiveness of convalescent plasma and hyperimmune immunoglobulin for the treatment of severe acute respiratory infections of viral etiology: a systematic review and exploratory meta-analysis. $J$ Infect Dis. 2015;211:80-90.

30. Duan K, Liu B, Li C, Zhang H, Yu T, Qu J, et al. Effectiveness of convalescent plasma therapy in severe COVID-19 patients. Proc Natl Acad Sci U S A. 2020;117:9490-6.

31. Mulangu S, Dodd LE, Davey RT Jr., Tshiani Mbaya O, Proschan M, Mukadi D, et al. A randomized, controlled trial of Ebola virus disease therapeutics. N Engl J Med. 2019;381:2293-303.

32. Lescure FX, Bouadma L, Nguyen D, Parisey M, Wicky PH, Behillil S, et al. Clinical and virological data of the first cases of COVID-19 in Europe: a case series. Lancet Infect Dis. 2020;20:697-706.

33. Wang Y, Zhang D, Du G, Du R, Zhao J, Jin Y, et al. Remdesivir in adults with severe COVID-19: a randomised, double-blind, placebo-controlled, multicentre trial. Lancet. 2020;395:1569-78.

34. U.S Food and Drug Administration. Investigational COVID-19 Convalescent Plasma-Emergency Indias; 2020. Available from: https:// www.fda.gov/vaccines-blood-biologics/investigational-new-drug-indor-device-exemption-ide-process-cber/investigational-covid-19-convalescent-plasma-emergency-inds\#foot. [Last accessed on 2020 Apr 04].

35. Stebbing J, Phelan A, Griffin I, Tucker C, Oechsle O, Smith D, et al COVID-19: combining antiviral and anti-inflammatory treatments. Lancet Infect Dis. 2020;20:400-2.

36. Horby P, Lim WS, Emberson J, Mafham M, Bell J, Linsell L, et al. Effect of Dexamethasone in Hospitalized Patients with COVID-19: Preliminary Report. New York: medRxiv; 2020.

37. Wang W, Tang J, Wei F. Updated understanding of the outbreak of 2019 novel coronavirus $(2019-\mathrm{nCoV})$ in Wuhan, China. J Med Virol. 2020;92:441-7.

38. World Health Organization. Coronavirus Disease 2019 (COVID-19) Situation Report-50. Geneva: World Health Organization; 2020. [cited 2 June 2020] Available from: https://www.who.int/emergencies/diseases/ novel-coronavirus-2019.

39. Munster VJ, Koopmans M, Van Doremalen N, Van Riel D, De Wit E. A novel coronavirus emerging in China-key questions for impact assessment. N Engl J Med. 2020;382:692-4. 